\title{
Nucleon-nucleon correlations probed at far sub-Coulomb energies
}

\author{
S. Szilner ${ }^{1, \star}$, L. Corradi ${ }^{2}$, D. Montanari ${ }^{3,4}$, T. Mijatović ${ }^{1}$, G. Pollarolo ${ }^{5}$, E. Fioretto ${ }^{2}$, A. Goasduff ${ }^{3}$, D. Jelavić \\ Malenica $^{1}$, D. Mengoni ${ }^{3}$, G. Montagnoli ${ }^{3}$, F. Scarlassara ${ }^{3}$, N. Soić ${ }^{1}$, A. M. Stefanini ${ }^{2}$, and J. J. Valiente-Dobón ${ }^{2}$ \\ ${ }^{1}$ Ruđer Bošković Institute, Zagreb, Croatia, \\ ${ }^{2}$ Istituto Nazionale di Fisica Nucleare, Laboratori Nazionali di Legnaro, Italy, \\ ${ }^{3}$ Universitá di Padova, and Istituto Nazionale di Fisica Nucleare, Padova, Italy, \\ ${ }^{4}$ IPHC, CNRS-IN2P3, Université de Strasbourg, Strasbourg, France, \\ ${ }^{5}$ Universitá di Torino, and Istituto Nazionale di Fisica Nucleare, Torino, Italy
}

\begin{abstract}
Excitation functions of one- and two-neutron transfer channels have been measured for the closed shell ${ }^{40} \mathrm{Ca}+{ }^{96} \mathrm{Zr}$ and superfluid ${ }^{60} \mathrm{Ni}+{ }^{116} \mathrm{Sn}$ systems from the Coulomb barrier energy to energies far below. By using the unique PRISMA performance in terms of resolution and efficiency, measurements at very low bombarding energies have been performed. In the case of the ${ }^{60} \mathrm{Ni}+{ }^{116} \mathrm{Sn}$ system, a complementary particle- $\gamma$ coincident measurements provide the ground and excited state populations.

The experimental transfer probabilities have been compared with semiclasical microscopic calculations that incorporate nucleon-nucleon correlations, together with known structure information of entrance and exit channels nuclei and reaction dynamics. These calculations well reproduce, in the whole energy range, one- and two-neutron transfer channels in shape and magnitude.
\end{abstract}

\section{Introduction}

Pairing interaction induces particle-particle correlations that are essential in defining the properties of finite quantum many body systems in their ground and neighbouring states. These structure properties may influence in a significant way the evolution of the collision of two nuclei, i.e. the effects of pair correlations affect a variety of nuclear processes. In this sense, low energy transfer reaction is one of the most important tool used to probe nucleonnucleon correlations in nuclear systems [1].

The pair correlations have been studied in measurements of two-particle transfer reactions by extraction of enhancement coefficients, defined as the ratio of the actual cross section to the prediction of models using uncorrelated states. Such enhancement factors, in principle, should provide a direct measurement of the correlation of the populated states. Unfortunately, the experimental evidence obtained by these factors is often obscured by the fact that almost all existing studies involve inclusive cross sections at energies higher than the Coulomb barrier, where the reaction mechanism is complicated by the interplay between nuclear and Coulomb trajectories.

On the contrary, at energies below the Coulomb barrier nuclei interact at very large distances, thus the interacting nuclei are only slightly influenced by the nuclear potential and $Q$ values are restricted to few $\mathrm{MeV}$ for the open transfer channels. These conditions diminish the complexity of calculations where only the overlap of the tails of

\footnotetext{
^e-mail: szilner@irb.hr
}

the intrinsic wave functions that are involved in the transfer process have to be taken into account. This energy regime is, on the other hand, characterized by low transfer cross sections. Only with the advent of large solid angle magnetic spectrometers the detection efficiency and resolution to identify the fragments in the heavy-ion collisions reached a sufficient level to make these experiments feasible [2-5].

In this work we present the results of a sub-barrier transfer measurements performed with the PRISMA spectrometer. Transfer probabilities for the closed shell ${ }^{40} \mathrm{Ca}+{ }^{96} \mathrm{Zr}$ [6] and superfluid ${ }^{60} \mathrm{Ni}+{ }^{116} \mathrm{Sn}$ [7] systems have been recently measured from the Coulomb barrier energy to energies far below it. Transfer yield has been detected at the level of $10^{-4}$ with respect to the elastic channel. We also performed a particle- $\gamma$ coincidence experiment for the ${ }^{60} \mathrm{Ni}+{ }^{116} \mathrm{Sn}$ system in order to investigate the ground and excited state population [8]. By taking advantage of the high energy resolution of $\gamma$ detectors, such measurements allowed to define how the transfer strength is distributed over the single states.

\section{Sub-barrier measurements}

In direct kinematics the transfer probability at large distances of closest approach correspond to fragments that are scattered at forward angles. In this angular range the overwhelming yield of elastically scattered particles often prevents a good identification for isotopes close to the projectile. At energies below the barrier, measurements of 


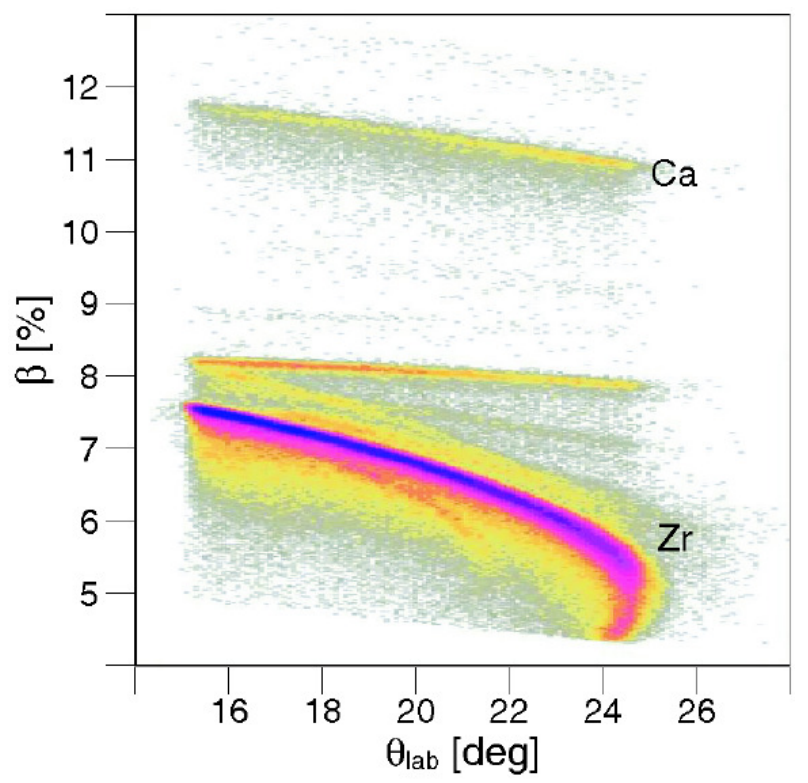

Figure 1. Velocity $(\beta=v / c)$ vs in-plane scattering angle $\left(\theta_{\mathrm{lab}}\right)$ for the reaction ${ }^{96} \mathrm{Zr}+{ }^{40} \mathrm{Ca}$ at $E_{\mathrm{lab}}\left({ }^{96} \mathrm{Zr}\right)=330 \mathrm{MeV}$. The magnetic fields of the spectrometer were set to bring the $\mathrm{Ca}$ ions with maximum yield near the center of the focal plane area. The events at large $\beta$ correspond to Ca-like recoils, and those at lower $\beta$ to $\mathrm{Zr}$ like ions scattered from $\mathrm{Ca}$, as well as from other elements in the target (from Ref. [6]).

heavy-ion-transfer reaction products present other significant technical difficulties. Backscattered ions have low kinetic energies which severely limit their identification. This can be partly overcome by the use of inverse kinematics, where the lighter target-like fragments are detected with a magnetic spectrometer at forward angles. In this case, the energy of the recoiled light fragment is usually large enough to obtain a good resolution in mass and nuclear charge.

In the measured reactions, ${ }^{96} \mathrm{Zr}+{ }^{40} \mathrm{Ca}$ and ${ }^{116} \mathrm{Sn}+{ }^{60} \mathrm{Ni}$, (lighter) target recoils have been detected with PRISMA, and the excitation functions at several bombarding energies have been obtained from the Coulomb barrier to 20$25 \%$ below, reaching $\sim 15.5 \mathrm{fm}$ of distance of closest approach. The identification of fragments has been done on an event-by-event basis by using, for the atomic number, the range of the ions as a function of the total energy released in the ionization chamber and, for the mass, by reconstructing the trajectories of the ions inside the magnetic elements of PRISMA [3, 9-12].

A fingerprint of the inverse kinematic is the behaviour of the velocity as a function of the focal plane position. Such matrix, the velocity vs in-plane scattering angle for the ${ }^{96} \mathrm{Zr}+{ }^{40} \mathrm{Ca}$ reaction, is displayed in Fig. 1. One sees the events corresponding to Ca-like recoils as well as those corresponding to $\mathrm{Zr}$-like ions. The ridges of these $\mathrm{Zr}$-like ions are due to scattering on $\mathrm{Ca}$ (main ridge presenting a bending around the kinematic limiting angle), on lighter elements (with smaller limiting angles), and on other heavier elements. The almost horizontal ridge, with high yield

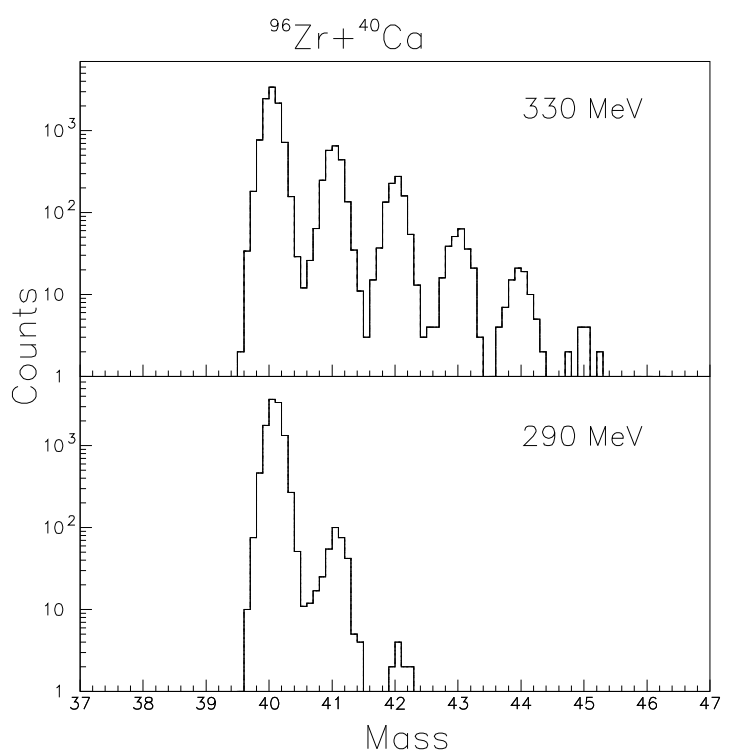

Figure 2. Mass distributions for pure neutron transfer channels obtained in the reaction ${ }^{96} \mathrm{Zr}+{ }^{40} \mathrm{Ca}$ at the indicated bombarding energies of the ${ }^{96} \mathrm{Zr}$ beam, i.e. $E_{\text {lab }}\left({ }^{96} \mathrm{Zr}\right)=330 \mathrm{MeV}$ (top) and $290 \mathrm{MeV}$ (bottom), and with the Ca-like recoils detected in PRISMA.

and with a $\beta \sim 0.08$, is due to $\mathrm{Zr}$ scattering in direct kinematics.

To illustrate the obtained mass resolution and the clear separation of different isotopes we show, as an example, the mass spectra at selected energies for the ${ }^{96} \mathrm{Zr}+{ }^{40} \mathrm{Ca}$ reaction in Fig. 2. At the higher energies, close to and above the Coulomb barrier, more than four neutrons have been transferred, while at the sub-barrier energies only one or at most two neutron transfers survive. Very similar mass distributions have been also observed for the ${ }^{116} \mathrm{Sn}+{ }^{60} \mathrm{Ni}$ reaction.

In order to have an overall description of the experimental data it is convenient to extract the transfer probability $P_{\text {tr }}$, defined as the ratio of the transfer cross section to the corresponding Rutherford cross section, and to plot these probabilities as a function of the distance of closest approach $D$ for a Coulomb trajectory. The extraction of the transfer probability $P_{\text {tr }}$ is rather alluring because the angular distributions obtained at different bombarding energies will be all superimposed, if the $Q$-value window does not change significantly. Further, the transfer probability at large distances $D$ may be obtained by measuring an excitation function at fixed center of mass angle or by measuring angular distributions at fixed energy. The transfer probabilities for the ${ }^{116} \mathrm{Sn}+{ }^{60} \mathrm{Ni}$ measurement are presented (full symbols), together with the calculations, in Fig. 3 for the $(1 n)$ and $(2 n)$ neutron transfer channels. While the transfer probabilities for the ${ }^{96} \mathrm{Zr}+{ }^{40} \mathrm{Ca}$ measurement are presented in Fig. 4.

To inspect in more details the distribution of the transfer strength we show in Figs. 5 and 6 the total kinetic energy loss (TKEL) distributions for the ${ }^{116} \mathrm{Sn}+{ }^{60} \mathrm{Ni}$ and ${ }^{96} \mathrm{Zr}+{ }^{40} \mathrm{Ca}$ reactions, respectively. The TKEL were con- 


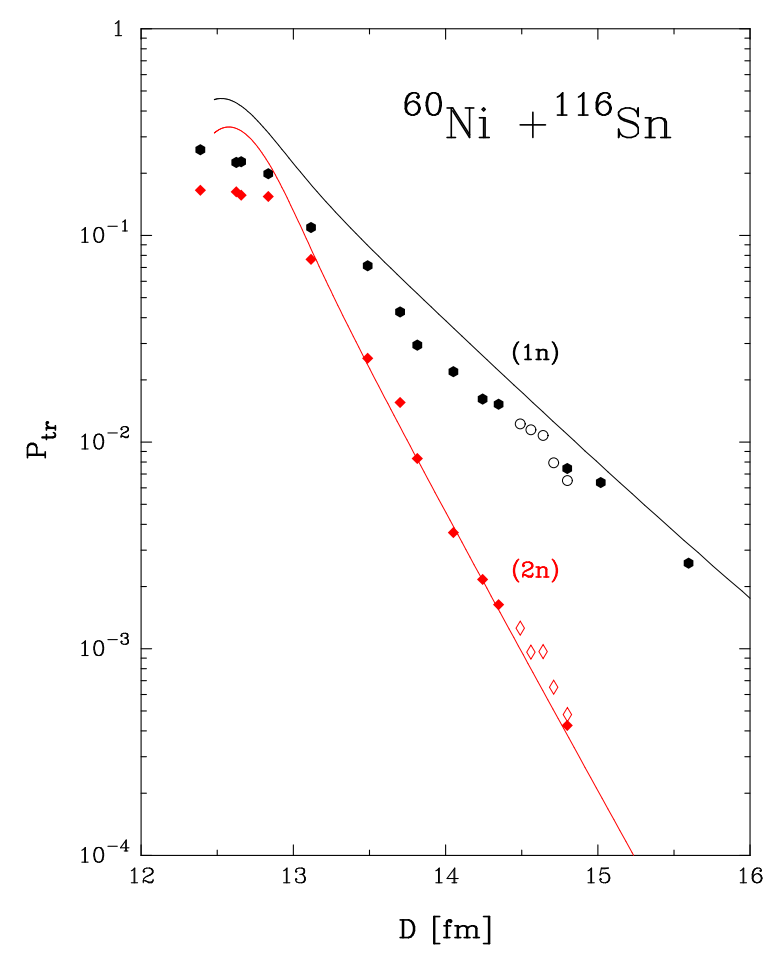

Figure 3. Experimental (points) and microscopically calculated (lines) transfer probabilities $P_{t r}$ as a function of the distance of closest approach $D$ for one- $\left({ }^{61} \mathrm{Ni}\right)$ and two-neutron $\left({ }^{62} \mathrm{Ni}\right)$ transfers in the ${ }^{60} \mathrm{Ni}+{ }^{116} \mathrm{Sn}$ system (from Ref. [8]).

structed assuming a binary reaction and imposing the conservation of momentum. The ground-state $Q$ values are indicated in figures. The width of the distributions is compatible with the intrinsic detector resolution, and the trajectory reconstruction, beam position and angle indetermination on target and target straggling effects.

In the case of the ${ }^{116} \mathrm{Sn}+{ }^{60} \mathrm{Ni}$ reaction, the experimental energy distribution for the elastic(+inelastic), one- and two-neutron transfer channels are concentrated around the ground state (see top panels of Fig. 5). In this very well $Q$-value matched system the reaction is dominated by the ground-to-ground state transition in $(0 n)$ and $(2 n)$ channels, and is close to the ground state in the $(1 n)$ channel. The situation is different in the ${ }^{96} \mathrm{Zr}+{ }^{40} \mathrm{Ca}$ reaction, in particular for the $(2 n)$ channel. One should notice that in this system for the $(2 n)$ channel the ground state $Q$-value is at +5.5 MeV. The TKEL spectra (see Fig. 6) display maxima at $\sim 6 \mathrm{MeV}$ at all measured energies, leaving the ground state unpopulated. These maxima, together with the known structure of the low energy states, suggest that in this channel the excited $0^{+}$states that were identified with the pair mode $[13,14]$ have been populated. Within the presently reached energy resolution, it is clear that a definite assignment of the populated single states was not feasible.

In general, with heavy ions, due to the illustrated limitation in the energy resolution with particle detectors, it is mandatory to combine such detectors with the gamma detection in order to distinguish the single states. Thus, we performed a particle-gamma coincidence experiment

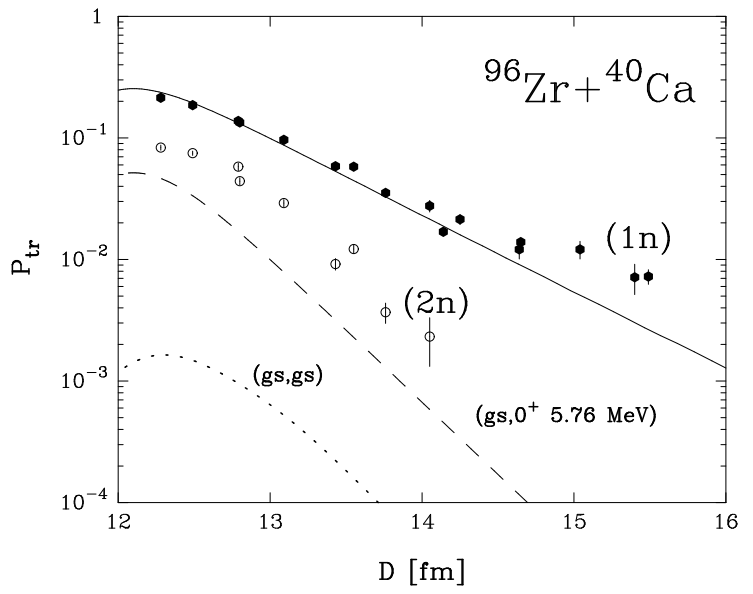

Figure 4. Experimental transfer probabilities $\left(P_{t r}\right)$ as a function of the distance of closest approach for the ${ }^{96} \mathrm{Zr}+{ }^{40} \mathrm{Ca}$ system. The $P_{t r}$ for one- and two-neutron transfer channels are represented with the full and empty symbols, respectively. The full line represents the inclusive transfer probability for one-neutron transfer, and the dotted line represents the calculation where only the ground-to-ground state transition for the two-neutron transfer has been included. For the dash line, the transition to the $0^{+}$excited state at $\sim 6 \mathrm{MeV}$ in ${ }^{42} \mathrm{Ca}$ has been included in the calculations (from Ref. [6]).

for the ${ }^{60} \mathrm{Ni}+{ }^{116} \mathrm{Sn}$ system by using the PRISMA spectrometer coupled to the Advanced Gamma Tracking Array (AGATA) demonstrator [15-17]. The demonstrator configuration included four triple cluster modules providing a simulated full-absorption efficiency of $2.64 \%$ for $1.3 \mathrm{MeV}$ $\gamma$ rays. This detector combination provides unique features for the selection of transfer products and the efficient detection of associated $\gamma$ rays.

\subsection{The particle- $\gamma$ coincident measurement}

We used a ${ }^{60} \mathrm{Ni}$ beam at $245 \mathrm{MeV}$ on the ${ }^{116} \mathrm{Sn}$ target, and detected the Ni-like fragments in PRISMA placed at $70^{\circ}$. This laboratory angle corresponds to large distances of closest approach, where the effect of absorption is negligible, with a still sufficient transfer yield. The transfer probabilities extracted from the angular distribution are shown (open symbols) in Fig. 3, demonstrating a consistent matching of the two experiments. In Fig. 5 we also report the TKEL (bottom) at distances comparable with the inverse kinematic measurement, where one can appreciate the similarity of the two distributions, which are clearly peaked around the ground state $Q$-value.

The particle- $\gamma$ coincidence measurement allowed to estimate the fraction of the total cross section going into excited states. In the inelastic channel the dominant transitions were from the $2^{+}$states of the binary partners (see Fig. 7). In the one-neutron transfer channel, a strong population of the low lying states of the single particle character has been observed, in particular the population of the $1 / 2^{-}$state in ${ }^{61} \mathrm{Ni}$ and of the $5 / 2^{+}$state in ${ }^{115} \mathrm{Sn}$. For the two-neutron transfer channel we observed only a few events compatible with the transition from the $2^{+}$state to 

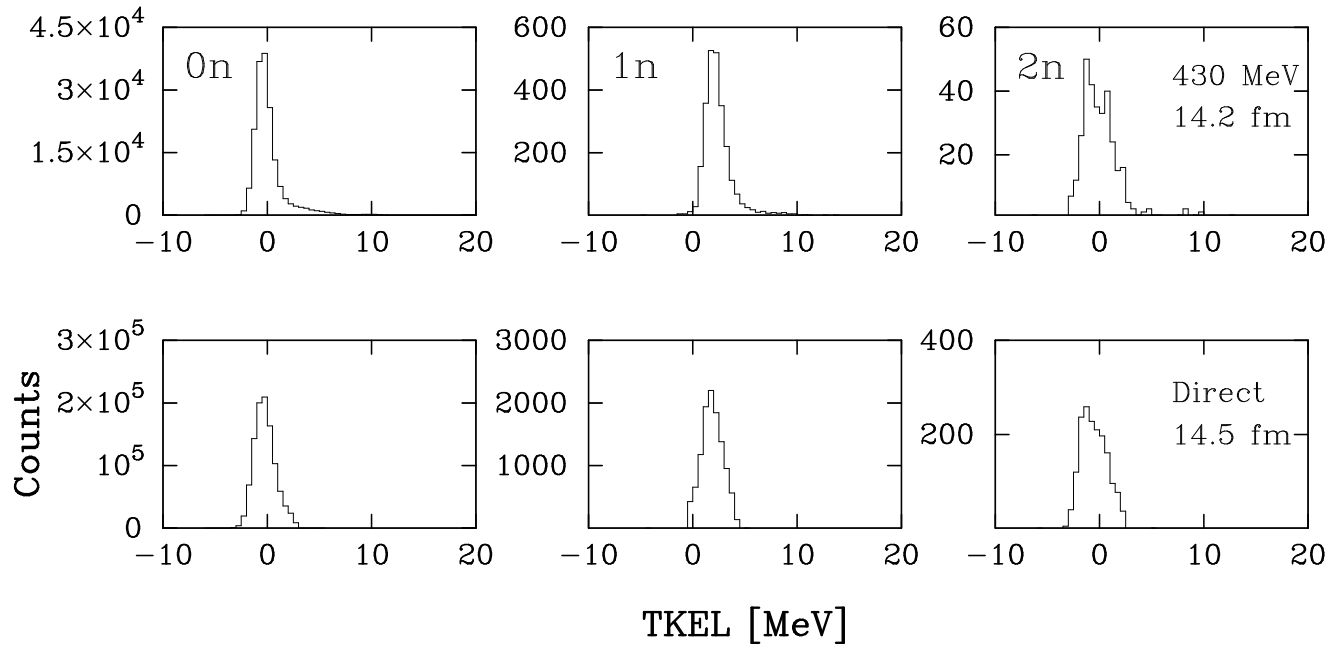

Figure 5. TKEL spectra obtained for the elastic(+inelastic) (0n), one- (1n) and two-neutron (2n) transfer channels at comparable distances of closest approach in the inverse kinematic (top) [7] and direct kinematic (bottom) [8] measurements for the ${ }^{60} \mathrm{Ni}+{ }^{116} \mathrm{Sn}$ system. The position of the ground state $Q$ values: $Q_{\mathrm{gs}}^{1 n}=-1.7 \mathrm{MeV}$ and $Q_{\mathrm{gs}}^{2 n}=+1.3 \mathrm{MeV}$.

the $0^{+}$ground state of ${ }^{62} \mathrm{Ni}$. For this two-neutron transfer channel, from the experimental intensities of the $\gamma$ lines, we concluded that the strength going to excited states is less than $24 \%$ of the total transfer strength of this channel. This finding demonstrates a dominance of the ground-toground state transition in this two-neutron transfer channel.

\section{Nucleon-nucleon correlations}

The transfer probabilities have been analyzed by employing a microscopic theory, which, for the first time in a heavy-ion collision, provided a consistent description of one- and two-neutron transfer channels, in shape and magnitude.

In more details, to compute the inelastic and oneneutron transfer channels the calculations have been performed by employing the same optical potential. Taking into account the collectivity of the low-lying states of the target and the projectile and the strong Coulomb field, a coupled-channels calculation have been performed for the inelastic channel by including the states which have been strongly populated in the particle- $\gamma$ measurement in the vibrational approximation and with the tabulated values for the deformation parameters. For the one-neutron transfer channel the calculations have been performed in the DWBA approximation. These calculations provide, in good approximation, the direct population of states which can be compared with the experimental intensities obtained in the particle- $\gamma$ measurement. Such comparisons provide a direct check on the one-particle form factors that have been used in the successive approximation for the two-neutron transfer. The transfer probability for the inclusive (1n) channel (full black lines in Figs. 3 and 4 for the ${ }^{60} \mathrm{Ni}+{ }^{116} \mathrm{Sn}$ and ${ }^{40} \mathrm{Ca}+{ }^{96} \mathrm{Zr}$ systems, respectively) has been calculated by summing over all possible transitions that can be constructed from the single particle states in projectile and target (see Refs. [6, 7]), and by weighting each transition with the corresponding spectroscopic factor. For both systems, the calculations agree well with the experimental transfer probabilities.

To calculate the ( $2 n$ ) channel one has to solve the wellknown system of semiclassical coupled equations up to the second-order Born approximation. The amplitude consists of three terms, the simultaneous transfer of the pair of nucleons, the nonorthogonality term, and the term which represents the successive process via an intermediate channel. For the calculation of the $(2 n)$ channel the groundto-ground state transition has been included following the formalism of Ref. [18]. In this formalism one can employ the same single particle form factors used for the (1n) channel. The ground states have been described in the BCS approximation with a standard state-independent pairing force.

For the (2n) channel of the ${ }^{60} \mathrm{Ni}+{ }^{116} \mathrm{Sn}$ system (see Fig. 3 ) the experimental points are very well described by the calculation. This fact indicates that the two-neutron transfer channel in this system is populating essentially only the ground state, in agreement with the experimental total kinetic energy loss distributions where the transfer strength was clearly concentrated around the ground state. Even more, the particle- $\gamma$ measurement provided the upper limit for the possible excited state population in the (2n) channel.

For the ${ }^{96} \mathrm{Zr}+{ }^{40} \mathrm{Ca}$ system [6] (see Fig. 4) the calculation describes well the one-neutron transfer channel, while in the case of the two-neutron channel the calculation which includes (dotted line) only the ground-toground state transitions underestimates the observed probabilities. Clearly, this transition does not contribute to the total transfer strength in agreement with what was experimentally observed in the TKEL spectra (see Fig. 6). As already discussed, the maxima of the TKEL for the (2n) channel have been connected with the population of the excited $0^{+}$states. Thus, in Fig. 4 with a dash line, we show the predicted transfer probability for the transition to 


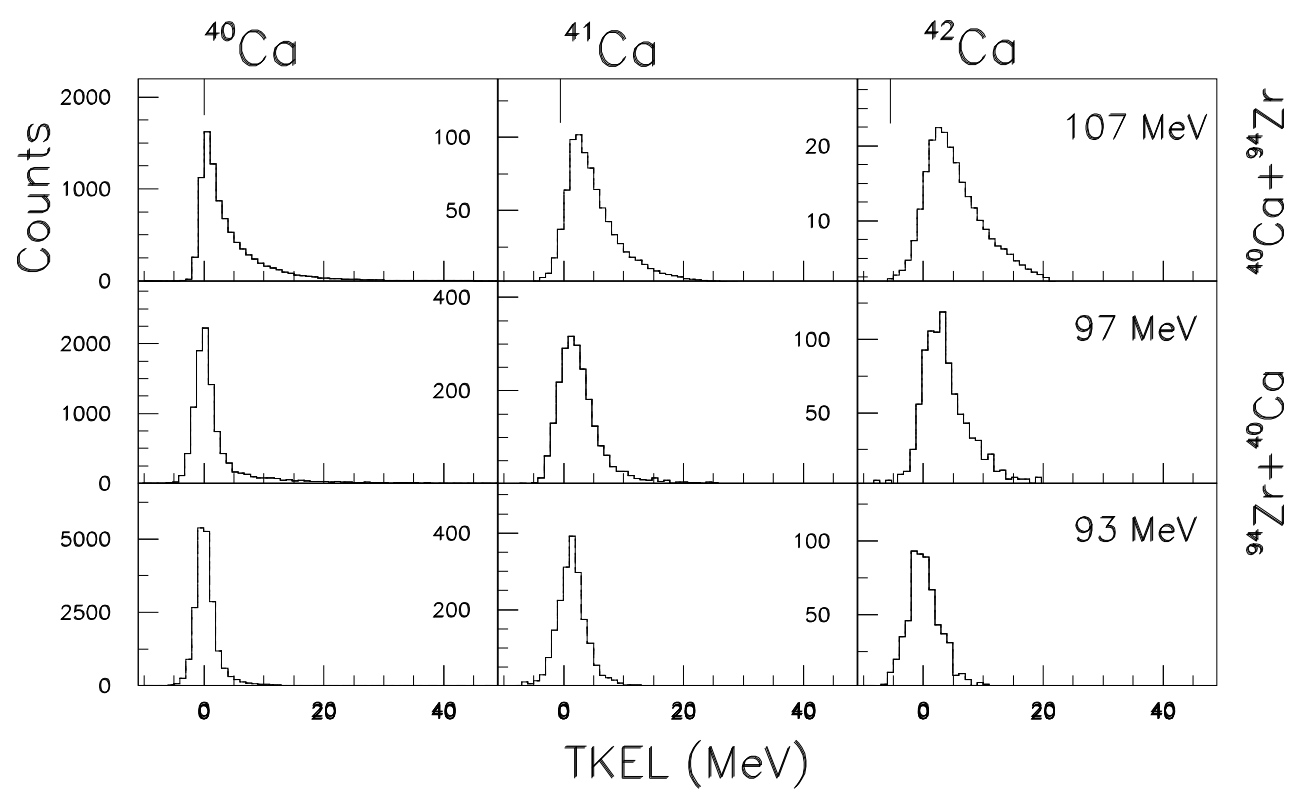

Figure 6. Experimental TKEL distributions for the elastic(+inelastic), one- and two-neutron transfer channels obtained in the "direct" ${ }^{40} \mathrm{Ca}+{ }^{96} \mathrm{Zr}$ [3] and inverse kinematic ${ }^{96} \mathrm{Zr}+{ }^{40} \mathrm{Ca}[6]$ measurements at the indicated center of mass energies. These energies correspond to about $10 \%$ above, at, and $10 \%$ below the Coulomb barrier. The vertical lines represent the ground state $Q$ values: $Q_{\mathrm{gs}}^{1 n}=+0.5 \mathrm{MeV}$ and $Q_{\mathrm{gs}}^{2 n}=+5.5 \mathrm{MeV}$.

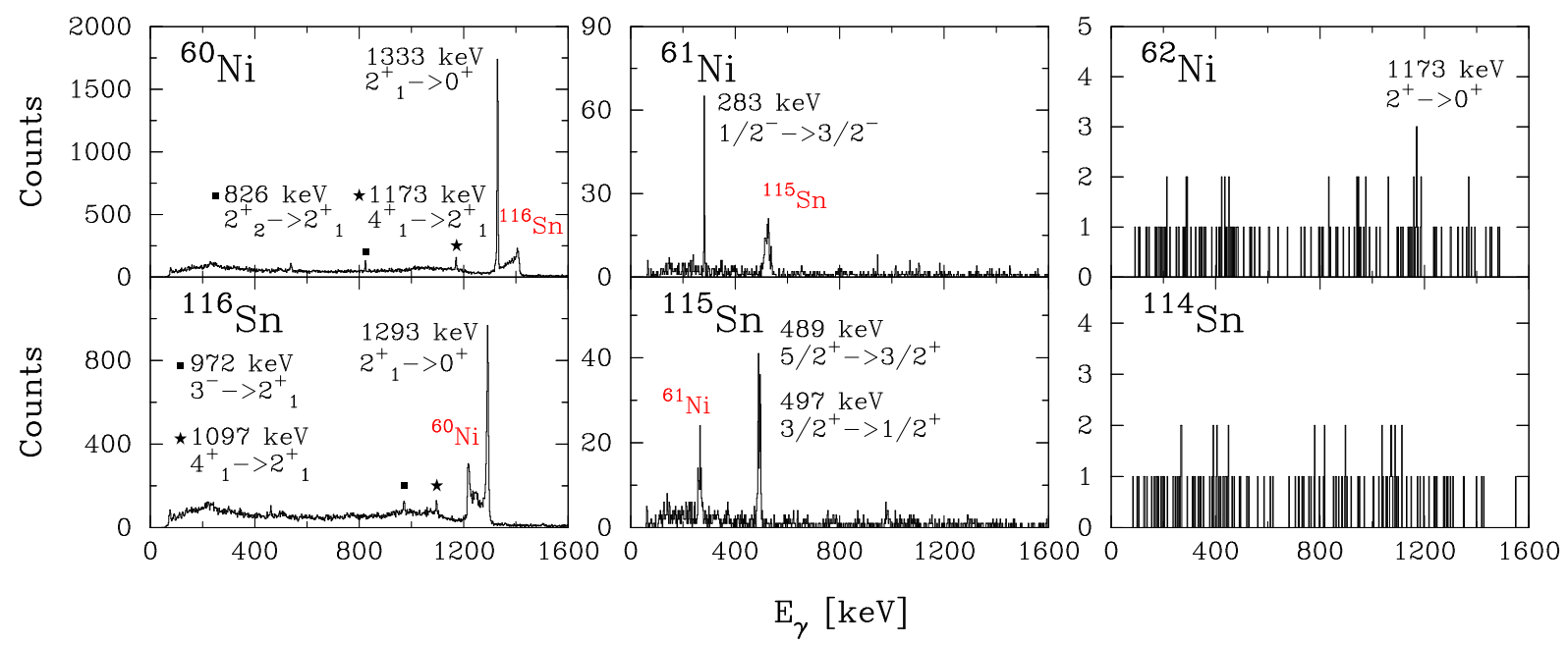

Figure 7. Top panels: Doppler-corrected $\gamma$ spectra for ${ }^{60} \mathrm{Ni},{ }^{61} \mathrm{Ni}$, and ${ }^{62} \mathrm{Ni}$ detected in PRISMA for the ${ }^{60} \mathrm{Ni}+{ }^{116} \mathrm{Sn}$ measured in the direct kinematic. Bottom panels: Doppler-corrected spectra for the heavy binary partners ${ }^{116} \mathrm{Sn},{ }^{115} \mathrm{Sn}$, and ${ }^{114} \mathrm{Sn}$. The strongest transitions are labeled with the $\gamma$-ray energy and spin and parity of the initial and final states. The broader peaks, corresponding to the wrongly Doppler-corrected reaction partner, are also labelled (red color) in each frame.

the $0^{+}$state at $\sim 6 \mathrm{MeV}$ in ${ }^{42} \mathrm{Ca}$. It is apparent that the contribution of this transition is much larger than the ground state one. Besides the good match with the $Q$-value, this is mostly due to the $2 p_{3 / 2}$ component that dominates its wave function and that has a much larger form factor. We stressed that the still missing strength was attributed to states with large angular momentum populated in the reaction, while only $0^{+}$to $0^{+}$transitions have been included in the theoretical calculation. At variance with the ${ }^{96} \mathrm{Zr}+{ }^{40} \mathrm{Ca}$ system, in the ${ }^{116} \mathrm{Sn}+{ }^{60} \mathrm{Ni}$ system the ground-to-ground state $Q$ values for one- and two-neutron transfer channels are very close to optimum $\left(Q_{\text {opt }} \sim 0\right)$, representing a beautiful $Q$-value matched case. One sees that in this case the employed microscopic theory very well reproduces the experimental data in all energy range, in particular the transfer probability for two neutrons is very well reproduced by considering solely the ground-to-ground state transition. It is very important to stress that the shown results are not 
coming from best fit procedure, but that they employed a microscopic theory that incorporates nucleon-nucleon correlations.

\section{Summary and outlook}

The advent of the large solid angle magnetic spectrometers, coupled to large $\gamma$ arrays, ensured significant advances in the field of multinucleon transfer reactions at energies close to the Coulomb barrier. We presented transfer probabilities for one- and two-neutron transfer channels in the ${ }^{40} \mathrm{Ca}+{ }^{96} \mathrm{Zr}$ and ${ }^{60} \mathrm{Ni}+{ }^{116} \mathrm{Sn}$ systems from the Coulomb barrier energy to energies corresponding to very large distances of closest approach where the nuclear absorption is negligible. The employed microscopic theory, that incorporates nucleon-nucleon correlations, very well reproduces the experimental data in the whole energy range, in particular, the transfer probability for two neutrons in the $Q$-value matched ${ }^{60} \mathrm{Ni}+{ }^{116} \mathrm{Sn}$ system is very well reproduced, in magnitude and slope, by considering solely the ground-to-ground state transition. It is very important to continue these studies, and to compare the experimental observables with microscopic calculations where nucleonnucleon correlations are treated together with the relevant structure and dynamics information.

As a follow up of these results, it will be important to study whether and to what extent the effect of neutron-neutron correlations, in the evolution of the reaction, is modified in the presence of high Coulomb fields. In the very heavy-ion collisions, the population of final states with high excitation energies and large angular momenta may significantly change the transfer strength of the ground-to-ground state transitions.

In this context, it is also important to investigate the role played by proton-proton and neutron-proton correlations. As known, multinucleon transfer reactions allow the transfer of large number of nucleons, and in order to study proton-neutron correlation one has to use systems where the population of the $( \pm n p)$ and $( \pm 2 p)$ channels are allowed by the $Q$-value considerations.

The issue connected with the pair correlations is presently at the focus of a renewal of interest [19-23], in particular in ongoing research with radioactive beams, where the pairing interaction may be significantly modified in nuclei with extended neutron distributions [24].
This work was partly supported by the EC FP7 ENSAR (262010), by Croatian Science Foundation (project 7194), and by the Scientific center of excellence for advance materials and sensors.

\section{References}

[1] D. M. Brink and R. A. Broglia, Nuclear Superfluidity: Pairing in Finite Systems (Cambridge University Press, Cambridge, England, 2005).

[2] L. Corradi, G. Pollarolo, and S. Szilner, J. of Phys. G 36, 113101 (2009).

[3] S. Szilner et al., Phys. Rev. C 74, 024604 (2007).

[4] H. Savajols and the VAMOS collaboration, Nucl. Phys. A 654, 1027c (1999).

[5] A. Cunsolo et al., Nucl. Instr. Methods Phys. Res. A 481, 48 (2002).

[6] L. Corradi et al., Phys. Rev. C 84, 034603 (2011).

[7] D. Montanari et al., Phys. Rev. Lett. 113, 052501 (2014).

[8] D. Montanari et al., Phys. Rev. C 93, 054623 (2016).

[9] T. Mijatović et al., Phys. Rev. C 94, 064616 (2016).

[10] D. Montanari et al., Eur. Phys. J. A 47, 4 (2011).

[11] L. Corradi et al., Nucl. Instrum. Methods Phys. Res. Sect. B 317, 743 (2013).

[12] T. Mijatović et al., Eur. Phys. J. A 52, 113 (2016).

[13] S. Szilner et al., Eur. Phys. J. A 21, 87 (2004).

[14] R.A. Broglia, O. Hansen and C. Riedel, Advances in Nuclear Physics, edited by M. Baranger and E. Vogt, Plenum, New York, 1973, Vol. 6.

[15] A. Gadea et al., Nucl. Instrum. Methods Phys. Res., Sect. A 654, 88 (2011).

[16] E. Farnea et al., Nucl. Instrum. Methods Phys. Res., Sect. A 621, 331 (2010).

[17] S. Akkoyun et al., Nucl. Instrum. Methods Phys. Res., Sect. A 668, 26 (2012).

[18] J. H. Sorensen and A. Winther, Nucl. Phys. A 550 (1992) 306.

[19] C. Simenel, Phys. Rev. Lett. 105, 192701 (2010).

[20] G. Potel et al., Phys. Rev. Lett. 105, 172502 (2010).

[21] G. Potel et al., Phys. Rev. Lett. 107, 092501 (2011).

[22] A. Lemasson et al., Phys. Lett. B 697, 454 (2011).

[23] G. Scamps and D. Lacroix, Phys. Rev. C 87, 014605 (2013).

[24] J. Dobaczewski et al., Phys. Rev. Lett. 72, 981 (1994). 